\title{
DISCHARGES, SPACE CHARGE, AND THE SHAPE OF ELECTRICAL TREES.
}

\author{
K.S. Bromley, L.A. Dissado and J.C. Fothergill \\ Electrical Power Engineering Group, \\ Department of Engineering, Leicester University \\ University Road, Leicester, LE1 7RH, UK
}

\section{INTRODUCTION}

The shape of electrical trees is critical in determining the life of electrical insulation subject to this type of degradation. For example a branch tree may lead to breakdown sooner than a bush tree which may be formed at a higher voltage, Densley [1]. This paper identifies a physical basis for determining tree shape.

The general features of the relationship between electrical tree propagation and partial discharges in tree tubules are well established, Dissado and Fothergill [2]. Discharges which originate at the initiating point electrode and travel to the tree periphery are associated with tip extension and occur during branch tree formation. On the other hand discharges restricted to the body of the tree widen existing tubules and promote bush-tree formation. The Discharge-Avalanche (D-A) Model, Dissado and Sweeney [3], of electrical tree propagation embodies the concept of discharge generated damage implicit in these observations. In the model it is assumed that discharges in tubules raise local fields in the polymer around the discharge path sufficiently to cause damage-generating avalanches in the polymer. Accumulation of the damage over many cycles leads eventually to the formation of a new tubule which is itself capable of sustaining a discharge, and hence constitutes an extension to the tree. By assuming that the average "quantity" of damage per one-electron avalanche is proportional to the number of ionisations generated, the D-A model can be expressed quantitatively in terms of the local electric field and a small number of material parameters. The expression for local damage derived in this way has a superexponential dependence on the local electric field along the avalanche path; this has been shown to be in accordance with experimental observations, Fothergill et al [4].

The values of the local fields are a combination of the divergent Laplace (i.e. no space charge) field of the point-plane electrode arrangement and the space-charge fields produced by discharge-deposited charges and avalanche rearrangement. However, the charge arrangement around the tubules also influences whether the discharge will initiate and its range of propagation; Bromley et al [5], Kaneiwa et al [6]. Thus the location and directions of greatest damage are linked with the discharge range and magnitude synergistically, Dissado et al [7]. In order to calculate tree propagation and shape without arbitrary assumptions regarding the magnitudes of the local fields, it is therefore necessary to derive their self-regulatory nature from a-priori calculations of the space-charge contributions, Dissado et al [7].

\section{DESCRIPTION OF DISCHARGES}

In early theoretical work, Fothergill et al [4], discharges in the tree tubules were taken to behave as a conducting extension of the point-electrode of nano-second duration. It was determined that the generation of fractal tree structures required the existence of time-dependent field fluctuations which were assumed to arise from the space-charge contributions, Dissado et al [7]. The discharges were assumed to extend throughout the tree. Bush structures were only formed when the fluctuation range diminished with increasing local Laplace field. Treating the discharges more realistically as an ionisation mechanism in the gaseous contents of the tubule which separates positive ions and electrons along the tubules in the form of a dipole, resulted in bush-type structures. Here positive charge was assumed to be adsorbed onto the tube walls predominantly towards the end of each tubule segment (assumed to be $10 \mu \mathrm{m}$ long), and the negative charge was assumed to penetrate the polymer. The space-charge field contribution tended to equalise the fields in transverse and axial directions at every tubule junction, leading to near equal growth in those directions at each potential extension site. These calculations, however, scanned the tree structure randomly for the possibility of essentially independent tubule-segment discharges at the peak voltages and therefore did not allow for long range discharge sequences that are a prominent feature of branch-type trees.

Recent calculations, Bromley et al [5], using a simulacrum of a tree built up from point charges, have shown that if the discharge deposits positive wall charge close to the negative charge at the tubule junctions the discharges will tend to be independent, i.e. of short range. The directions of local fields will not be governed 


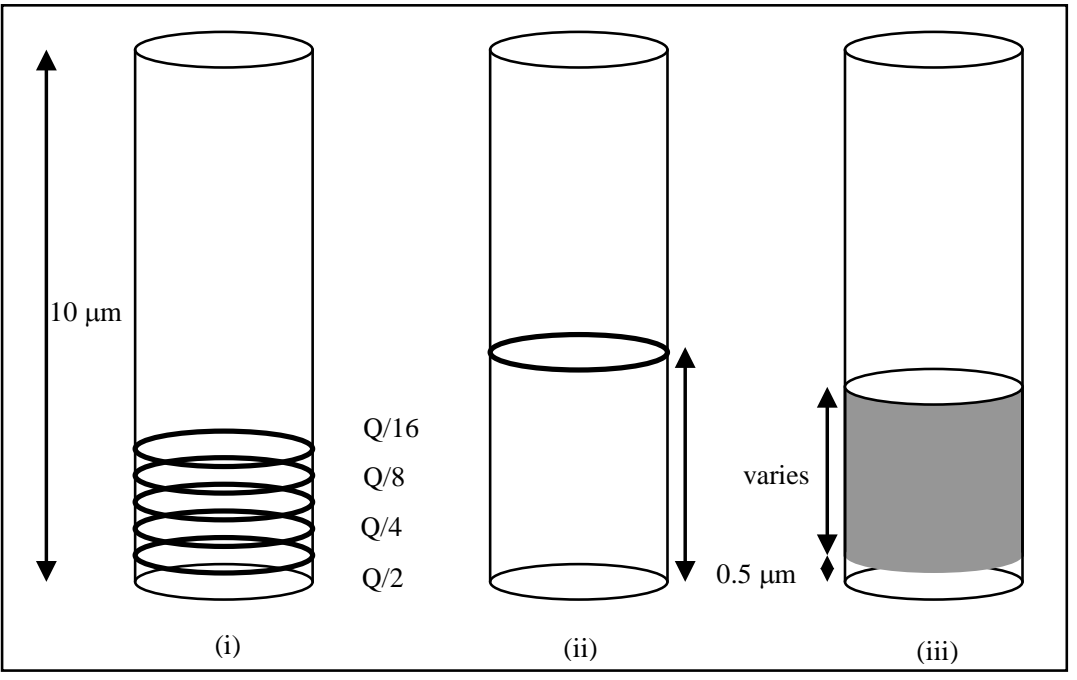

Fig. 1: Annuli of positive wall charge in three arrangements along the tubule

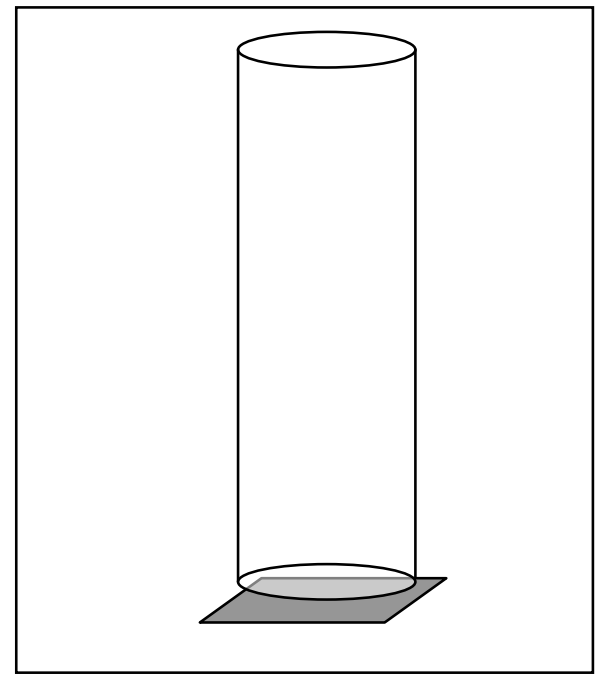

Fig. 2: Plate of negative charge at tubule tip at tip of tree.

by the Laplace field direction unless very close to the point electrode and so, at every site where tubules join, damage will be produced in all directions. On the other hand, when the positive wall charge is displaced substantially back along the tubule, the discharge would tend to extend over a long range, giving a large field increase at the tree tips where the discharge terminated and very little at intermediate sites. A hidden feedback effect was also found, with branch-structures promoting the initiation of long range discharges from the pointelectrode in comparison to bush-structures. These results identify the way in which long-range discharges serve to produce branch-shapes whereas independent discharges produce bush-shapes.

The existence of sequences of discharges is related to the range of the positive wall-charge displacement. They also give an insight as to the differences between very narrow $(1 \mu \mathrm{m})$ and wider $(50 \mu \mathrm{m})$ tubules since, in the latter case, the positive charge should be less easily trapped on the tubule walls and tend to support longer range discharges.

\section{PRESENT CALCULATIONS}

Here we remove the restriction to point charges in our description of space-charge fields. The negative charge penetrating the polymer is represented by a plate of charge, and the positive wall charge by annuli of charges which may be spaced along the tubule or widened, in order to represent the different ways in which wall charge may accumulate and displace. The arrangements of wall charge considered are shown in Fig. 1., and represent three different physical situations.

i) The positive charge is adsorbed close to where it is produced giving an exponentially increasing charge density along the tube. This is modelled as a set of annuli each of geometrically increasing charge spaced at $0.5 \mu \mathrm{m}$ (typical ionisation path length);

ii) Positive charge is adsorbed close to where the last ionisation in the gas takes place, and may then displace along the tubule back towards the point electrode, and

iii) The positive charge aggregates on the walls in the form of a wide cylinder of constant charge density extending a substantial fraction of the tube length.

For each of these three arrangements we evaluate the fields for the following situations. Firstly we consider the tubule to end at a tree tip and we determine axial and transverse fields in the polymer. In this case we treat the negative charge in the polymer as a plate of charge situated just below the tree tip, Fig 2. Although this is something of an approximation, other more complex and asymmetrical charge distributions can be built up using charge plates as components. These calculations are intended to determine which of the charge arrangements is most effective at promoting axial growth in favour of transverse growth and hence bifurcation at the tree tip.

Secondly we consider a tubule which links two other tubules, one proximal to the point electrode and one distal, to form an extended tubule. The negative charge in each tubule is considered to form an annulus around each of the joints of the tubules. The field is calculated a) along the middle tubule, b) along the distal tubule, and c) in the polymer around the tubule in question, see Fig. 3. In practice the three tubule segments are unlikely to be precisely in line as shown and so discharges are likely to continue to be confined to the individual segments after they have joined together.

In all cases we consider the effect of a previous negative discharge in depositing charge in the other tubules and represent that charge by positive and negative annuli. The purpose of this calculation is threefold. Firstly we 


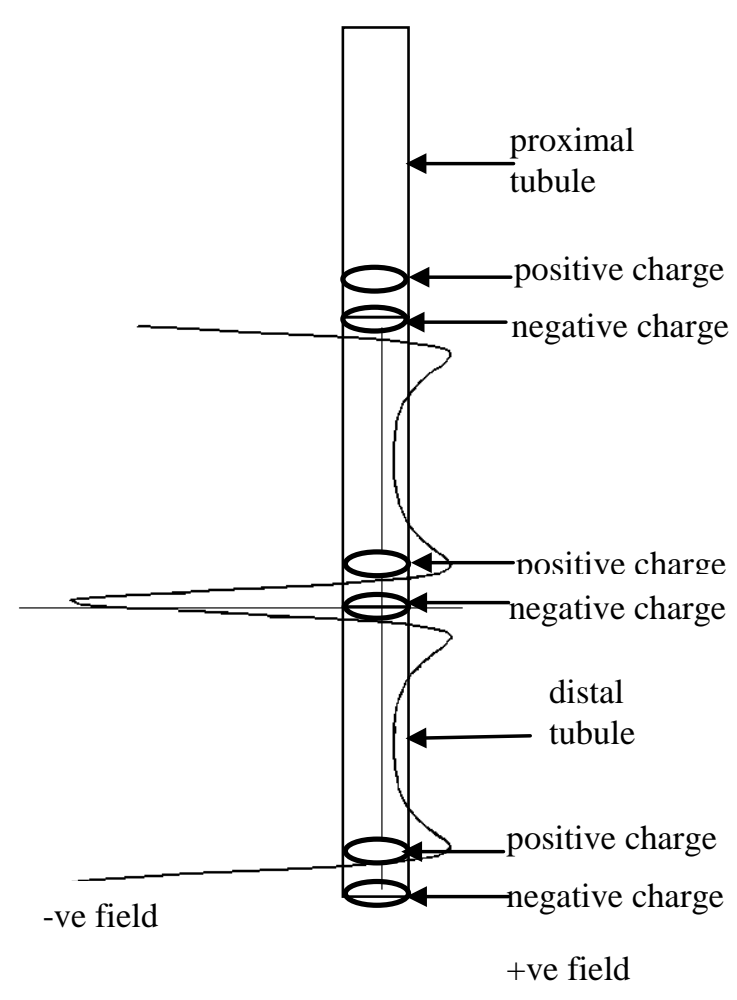

Fig 3 Extended tubule

estimate the magnitude of the avalanche-generating fields to the sides of the tubule in the various charge arrangements so as to determine which situation is most likely to lead to damage. Secondly we determine which arrangement is most likely to enable the discharge to continue into the next tubule, and thirdly we determine the arrangement that will extinguish continuance of the discharge within the central tubule, Kaneiwa [6]. In this way the range of the discharge can be related to its damage generating capability both at the tree tips and along its path, and to the arrangement of its charge deposition, in particular the amount of displacement of positive charge towards the point electrode. This latter point potentially relates discharge types and sites of damage to tube diameters and the production of weakly conducting tube walls as the tree grows, Bromley et al [5].

\section{RESULTS}

Potentials have been calculated at points on a $10 \mu \mathrm{m}$ grid. In the following, the origin is defined as the base of the single tubule or central tubule segment, i.e. the end of the tubule at which the discharge ends. The potential difference is calculated as the potential at the grid point in question minus that at the origin. The potential difference is proportional to the total positive or negative charge produced during a discharge and hence is expressed in $\mathrm{V} / \mathrm{fC}$ (i.e. $10^{-15} \mathrm{~V} / \mathrm{C}$ ). Reported fields $(\mathrm{kV} / \mathrm{mm} / \mathrm{fC})$ are calculated by dividing potential differences between adjacent grid points by $10 \mu \mathrm{m}$, they therefore represent averages over that distance.

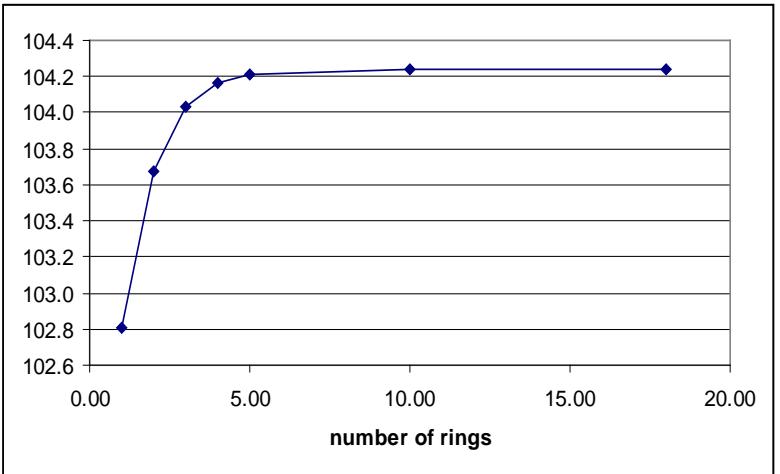

Fig. 4(i): Variation of potential difference (V/fC) between tubule ends due to annuli of exponentially increasing positive charge (Fig. 1(i)), and plate of negative charge, (Fig. 2)

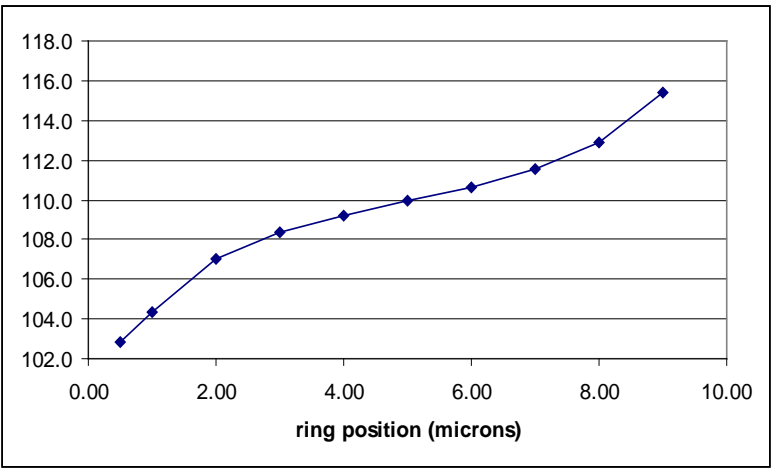

Fig. 4(ii): Variation of potential difference (V/fC) between tubule ends with single annulus of positive charge (Fig. 1(ii)), and plate of negative charge, (Fig. 2)

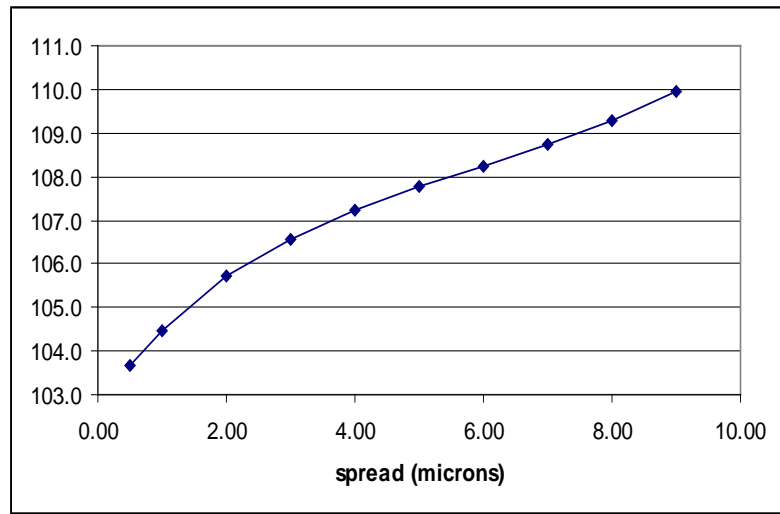

Fig. 4(iii): Variation of potential difference (V/fC) between tubule ends with spread of cylinder of positive charge (fig. 1(iii)), and plate of negative charge, (fig. 2)

\section{Single Tube at Tree Tip}

For the three arrangements of positive space charge shown in Fig. 1 with the negative charge plate shown in Fig. 2 the potential differences across the tubule has been calculated and are shown in Figs. 4 (i)-(iii). 
Increasing the number of annuli of exponentiallyincreasing charge rapidly leads to an asymptotic limit on the potential difference of $104.4 \mathrm{~V} / \mathrm{fC}$ as the bulk of the charge is still deposited nearest to the tip of the tubule, Fig. 4(i). The charge centroid moves from $0.5 \mu \mathrm{m}$ towards $1.0 \mu \mathrm{m}$ as the number of annuli increases. With 1 annulus at $1 \mu \mathrm{m}$ (Fig. 4(ii)) a very similar potential difference is found. The more complicated multi-annuli structure of Fig. 4(i) can therefore usually be replaced by the single-annulus structure of Fig. 4(ii) gaining an increase in computation speed without loss of significant precision.

As the annulus position moves back up the tubule, Fig. 4(ii), or the spread of the cylinder of positive charge increases along the tubule, Fig. 4(iii), the potential difference becomes more positive and therefore inhibits a further immediate discharge until the positive charge has dissipated. Whether the annulus of charge moves along the tube wall, as in arrangement ii) or spreads out, as in arrangement iii) depends on various physical parameters, such as the type of polymer, the 'age' of the tubule and may vary over the AC cycle. The model of tree growth under development here is intended to predict the shape of the tree grown under specific conditions. It is therefore of interest to consider the influence that these different wall charge arrangements have on the likelihood of the tree developing a bush shape or a branch shape.

By adding together the potential difference due to each arrangement of positive charge and that due to the plate of negative charge (maintaining charge neutrality in each case) the ratio of the transverse and axial fields in the polymer at the tip of the tree can be calculated and hence whether the tubule is more like to extend to the sides or continue in the present direction. Typical average fields per fC of charge ( 1 annulus, $0.5 \mu \mathrm{m}$ back) are:

- transverse field in polymer at tree tip: $1.8 \mathrm{kV} / \mathrm{mm}$

- axial field in polymer ahead of tree: $4.5 \mathrm{kV} / \mathrm{mm}$

- within tubule: $10 \mathrm{kV} / \mathrm{mm}$

Fig. 5 shows that this ratio only varies from 2.25 to 2.45. In the case of the annulus of charge moving back along the tubule, or spreading out in a cylinder along the tubule the ratio decreases slightly and therefore the growth to the side becomes more likely. Even though this is a slight change it should be remembered that the rate of damage is a super-exponential function of field.

If more plates of negative charge are added further into the polymer (to represent further electron injection and avalanching), these tend to equalise the axial and transverse fields making bush tree growth more favourable. Contributions to the local field from the applied field have a similar ratio and have been

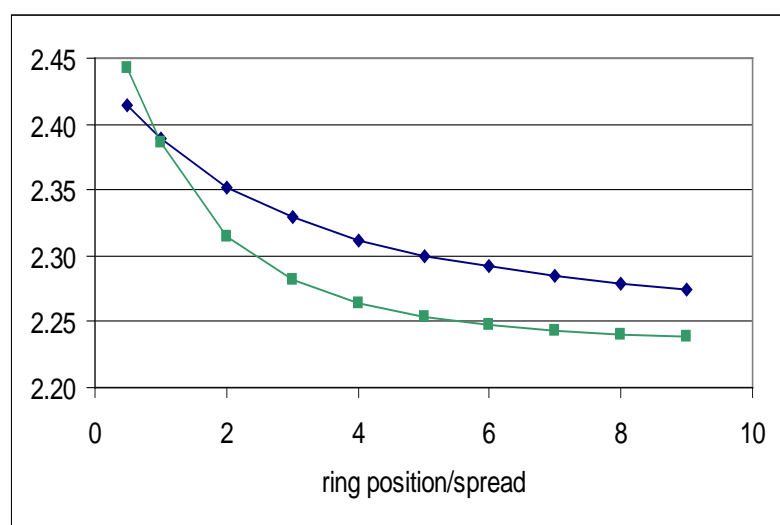

Fig. 5: Variation of axial/transverse field ratio in polymer ahead of tree tip for positive space charge arrangements shown in Fig. 1(ii) and (iii).

neglected here as has the field due to charge in the rest of the tree.

\section{Extended Tubules within Tree Structure}

The extended tubule, comprising the three linked tubule segments, and the axial field distribution due to the space charges are shown in Fig. 3. The potential differences in the three segments and the field in the adjoining polymer are now found to be highly dependent on the charges deposited on the tube walls. Table 1 shows, per fC of charge at each annulus:

- the transverse field $(\mathrm{kV} / \mathrm{mm})$ in the polymer at the join of the middle and distal segments. This field may produce damage in the polymer which may cause a new tubule to grow orthogonally at this point.

- the potential difference (in volts) across the $(10 \mu \mathrm{m})$ distal tubule segment. A positive value promotes discharging.

- the potential difference (in volts) across the $(10 \mu \mathrm{m})$ middle segment. A negative value promotes discharging (as the origin is at the join). Calculations (omitted here for simplicity) showed that positive charge spread as a cylinder back along the tube lead to similar conclusions.

These values have been calculated for three cases:

i. The first case is as shown in Fig 3. There are annuli of negative charge at the ends of the discharges assumed to have taken place in each tubule segment. Overall electrical neutrality is maintained by positive charge annuli located $0.5 \mu \mathrm{m}$ before the end of the discharge.

ii. For the second case it is assumed that another discharge has taken place in the middle segment. The annuli of negative and positive charge at the 
end of this discharge have therefore doubled in value of charge.

iii. In the third case it is postulated that the positive charge is more mobile and able to move back along the wall of the tubule. The situation is considered in which the positive annuli from the first discharge have moved back $6.0 \mu \mathrm{m}$.

Typical discharges in individual tubule segments may have values of approximately $10 \mathrm{fC}$; it may be appropriate to multiply the values in the table by approximately ten to give typical values.

It can be see that, in each case, a discharge in the middle tubule promotes a discharge in the next (distal) tubule. Thus we would normally expect a discharge near the "needle" to propagate towards the tip providing fields generated by other parts of the tree do not interfere with this process.

Table 1: Fields and potential differences after discharges in extended tubule (see text).

\begin{tabular}{|c|c|c|c|}
\hline & $\begin{array}{c}\text { transverse } \\
\text { field at } \\
\text { join } \\
\mathrm{kV} / \mathrm{mm} / \mathrm{fC}\end{array}$ & $\begin{array}{c}\text { p.d. } \\
\text { across } \\
\text { distal } \\
\text { tube } \\
\text { V/fC }\end{array}$ & $\begin{array}{c}\text { p.d. } \\
\text { across } \\
\text { middle } \\
\text { tube } \\
\mathrm{V} / \mathrm{fC}\end{array}$ \\
\hline $\begin{array}{c}\text { (i) initial discharge } \\
\text { (ii) } 2^{\text {nd }} \text { discharge, } \\
\text { lower mobility +ve. } \\
\text { charge }\end{array}$ & 0.37 & 8.2 & -0.5 \\
\hline $\begin{array}{c}\text { (iii) } 2^{\text {nd }} \text { discharge, } \\
\text { higher mobility +ve. } \\
\text { charge }\end{array}$ & 1.0 & 8.2 & 0.5 \\
\hline
\end{tabular}

A second discharge in the middle tubule increases the transverse field at the join. Although the values are significantly less than on the tree periphery they are still likely to cause significant damage over a period of time.

If the positive charge is slow to move back along the tubule wall then another immediate discharge is not inhibited and, provided the Laplace field is sufficiently high, several discharges along such extended tubules may take place in each half cycle.

If the positive charge is allowed to move back along the tubule wall then this strongly inhibits another immediate discharge in the same tubule; it is necessary to wait until the positive charge has diffused into the polymer. Depending on how fast this happens, it is likely that there will be only one discharge propagating along such extended tubule structures per half cycle.

\section{DISCUSSION}

The nature of the discharge within a tubule may have to be considered further. At present a discharge is considered in which (i) there is axial symmetry and (ii) electron multiplication and gas ionisation proceeds down the length of the tubule once the potential difference across the tube exceeds a certain value.

As an electron discharge proceeds, electrons are accelerated by the electric field increasing their kinetic energy. If an electron has sufficient kinetic energy on colliding with a gas molecule it may cause ionisation resulting in an extra free electron and a positivelycharged ion. During the time-scale of a discharge, the relatively-immobile positive ions will move much less than the electrons. Immediately after a discharge the tubule contains gas molecules and ions, the latter in an increasing concentration towards the end of the discharge. In a discharge unrestricted by tubule walls approximately half the positive gas ions will have been created one ionisation path length (of the order of $0.5 \mu \mathrm{m}$ in this case) from the end of the discharge. The discharge electrons will quickly enter the polymer. Due to their thermal energy and self-repulsion the positive ions will then move towards the tubule walls where they will be adsorbed. Whilst they remain on the walls and, because most are produced near the end of the discharge, such positive wall charges are likely to promote, at least to some extent, another discharge in that tubule. This behaviour was observed in the previous section.

It is unlikely that a discharge would initiate or therefore continue to be axially symmetric. In this case annuli will be produced which have an asymmetric charge distribution Instead of forcing a subsequent discharge trajectory towards the axis, as in the uniform charge distribution trajectory, this arrangement will distort the trajectory of the discharge away from the tubule axis; the tubule axis is likely to be a metastable trajectory for the discharge. If many discharges terminate on the tubule side wall rather than the end then this is likely to result in wall erosion and tubule widening. Since there is electrostatic repulsion both between and amongst (positive) discharge ions and positive wall charges, the wall charge density distribution may gradually become less uneven and "patchy" than might be thought by only considering only unstable discharges. We may therefore approach the situation represented by Fig. 1(iii), a cylinder of positive charge spreading along the tubule. The field distribution from such a cylinder, spread back over $8 \mu \mathrm{m}$ of the wall of a tubule at the tree periphery, is shown in Fig. 6. This figure considers only the effect of the positive charge, fields due to negative charges and 


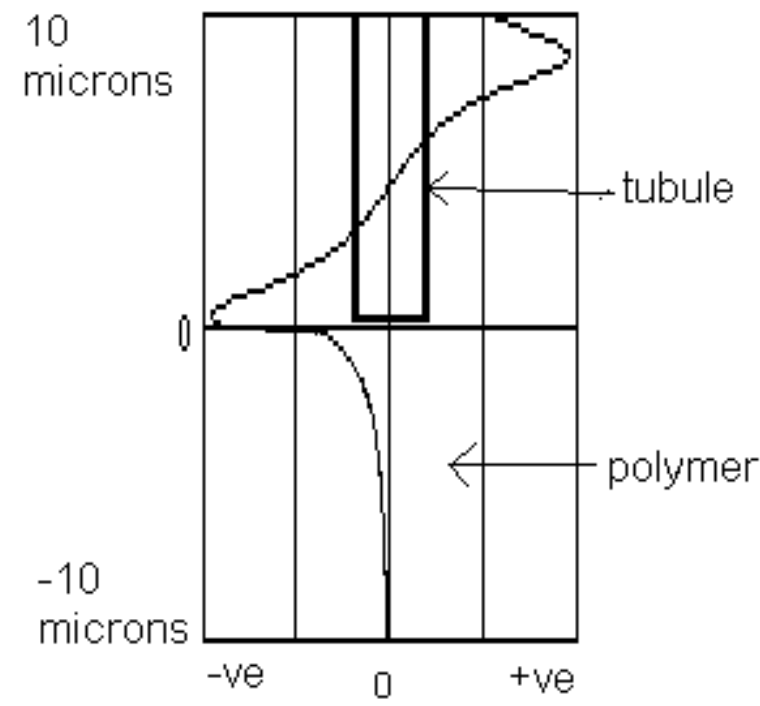

Fig. 6 Axial field, in the direction of the discharge along a tubule on the tree periphery and into the polymer ahead.

the Laplace (applied) field need to be added to give a true picture.

The positive contribution to the field at the top of Figure 6 would tend to further move the cylinder of positive charge up the tubule wall and would tend to promote discharging. The negative field in the bottom half of the tubule in this figure would also tend to spread the cylinder down the tubule. This is to be expected - the positive charge is self-repulsive. However this negative contribution to the field would tend to inhibit discharging. Any discharges in a tubule with such a charge distribution are therefore likely to terminate on the tubule wall and lead to tube widening or perhaps the formation of new branches .

\section{CONCLUSIONS}

The movement of residual positive charges along the walls of tree tubules and into the surrounding polymer is a critical determining factor in the shape (bush or branch) of electrical trees.

Positive charge that has a higher mobility along tubule walls promotes single discharges propagating to the tree tip. Lower mobility positive wall charge tends to more, but perhaps smaller, discharges. The former may favour branch tree growth, the latter bush trees.

Spread out positive wall charge may occur when there are a number of discharges per half cycle. The resulting damage may favour bifurcation (transverse extension).

\section{REFERENCES}

[1]. R.J.Densley "An investigation into the growth of electrical trees in XLPE cable insulation” IEEE Trans. Electr. Insul. 14 148-158, 1979

[2]. L.A. Dissado and J.C. Fothergill "Electrical Degradation and Breakdown in Polymers" $\mathrm{P}$. Peregrinus for IEE, London, 1992

[3]. L.A.Dissado and P.J.J. Sweeney, "Physical model for breakdown structures in solid dieletrics” Phys. Rev. B48, 16262-16268, 1993

[4]. J.C. Fothergill, L.A. Dissado, and P.J.J. Sweeney, "A Discharge-avalanche theory for the propagation of electrical trees. A physical basis for their voltage dependence” IEEE Trans. Diel. \& EI. 4, 474-486,1994

[5]. K.S. Bromley, L.A. Dissado, and J.C. Fothergill, "Local field calculations for electrical trees in point-plane geometry” Ann. Rep. CEIDP, 304-307, 1997

[6]. H. Kaneiwa, Y. Suzuoki, and T. Mizutani, "Partial discharge characteristics in an artifically-simulated tree channel” Ann. Rep. CEIDP, 317-320, 1997

[7]. L.A.Dissado, S.J. Dodd, J.V. Champion, P.I. Williams, and J.M. Alison, "Propagation of electrical tree structures in solid polymeric insulation” IEEE Trans. Diel. \& EI 4, 259-279, 1997

\section{ACKNOWLEDGEMENTS}

We gratefully acknowledge the support of EPSRC (GR/L/10589) and National Grid Company plc for this work. 